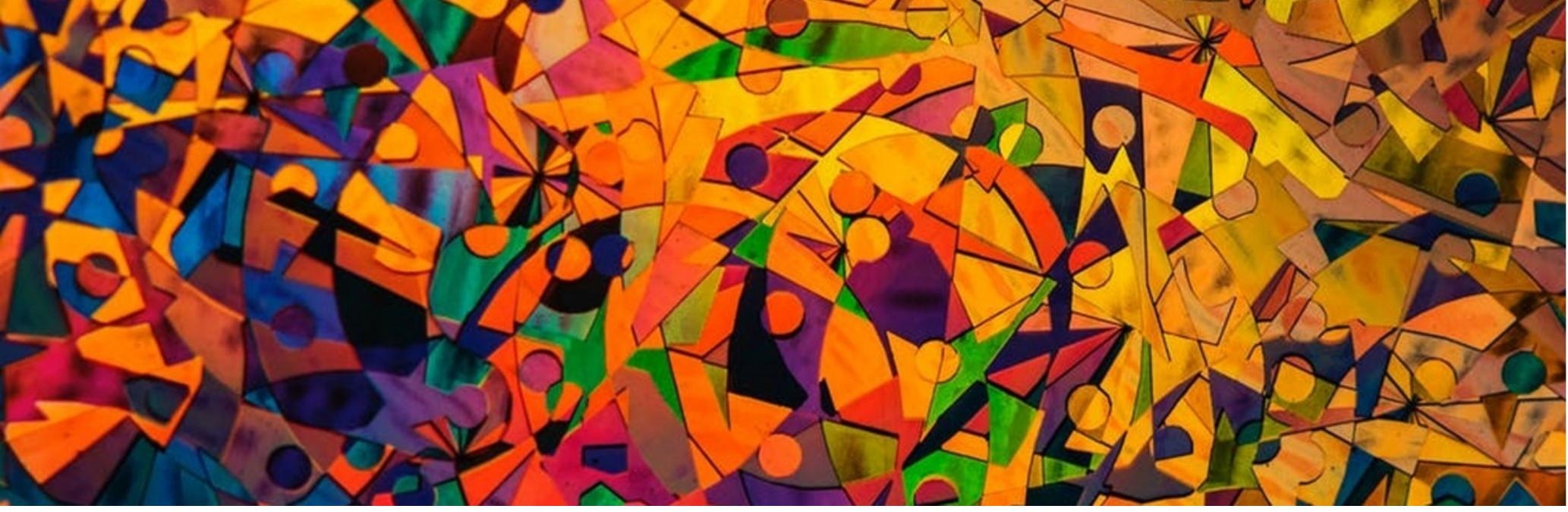

\title{
DEMETRIOS PRIZE 2019
}

$\begin{aligned} \text { Authors: } & \text { Roland Mertelsmann } \\ \text { Submitted: } & \text { 2. January } 2019 \\ \text { Published: } & 6 . \text { January } 2019 \\ \text { Volume: } & 6 \\ \text { Issue: } & 1 \\ \text { Affiliation: } & \text { Uniklinik Freiburg, Med } 1 \\ \text { Languages: } & \text { German } \\ \text { Keywords: } & \text { Demetrios, competition, Humanities, Sciences, Arts, Prize } 2019 \\ \text { Categories: } & \text { News and Views } \\ \text { DOl: } & 10.17160 \text { /josha.6.1.521 }\end{aligned}$

Abstract:

The JOSHA Team is very pleased to share with you information about our second Demetrios Prize 2019. For the categories BACHELOR, MASTER and DOCTORAL thesis the International Academy of Science, Humanities and Arts (IASHA e.V.) supported by the Biothera Foundation will select 3 theses in each category and award a prize of $€ 500$ each. Our editors will select the winners. The manuscripts of the winners will be published in the Journal of Science, Humanities, and Arts - JOSHA, with a unique DOI for each paper. Theses in either GERMAN, ENGLISH, FRENCH or SPANISH may be submitted. Bachelor, Master, and doctoral theses should be submitted by mail to admin@josha-archive.org.until June 15, 2019. The "Journal of Science, Humanities, and Arts - JOSHA" has been initiated to create a novel internet platform to access the broad diversity of important discoveries and creativity in the fields of Science, Humanities, and Arts. At JOSHA we believe, that "Knowledge that is not communicated is wasted knowledge"

\section{JOSHA Jommalosiencee Humanities and Arts




\section{DEMETRIOS PREIS 2019}

\section{Möchtest Du Deine Arbeit im JOSHA JOURNAL publizieren?}

JOSHA als Open Access Library, die Naturwissenschaftlern, Geisteswissenschaftlern und Künstlern in allen Ländern in allen Sprachen zur Verfügung steht, ist stolz darauf, Ihnen den Demeterios - Preis vorstellen zu dürfen.

Für die Kategorien BACHELOR-, MASTER- und DOKTORArbeiten wird die

International Academy of Science, Humanities and Arts (IASHA e.V.) mit Unterstützung der Biothera Stiftung jeweils 3 Preise in Höhe von 500 Euro vergeben. Unsere Editoren werden die Auswahl treffen und die Arbeiten der Gewinner werden mit eigener DOI Nummer in der online Zeitschrift publiziert!

Bachelor-, Master- und Doktor-Arbeiten könnt Ihr uns ab sofort an admin@joshaarchive.org zukommen lassen.

Bewerbungsfrist bis 15. Juni 2019

Das JOSHA-Team freut sich sehr auf Eure Bewerbungen!

www.josha-journal.org

admin@josha-archive.org

Internationale Akademie der Wissenschaften

und Künste e.V.

www.iasha.org 


\section{JOSHA JOURNAL}

Die International Academy of Sciences, Humanities and Arts, IASHA, ist Herausgeber des Journal of Sciences, Humanities and Arts - JOSHA.

JOSHA ist eine offene, multidisziplinäre und mehrsprachige Zeitschrift für den Zugang zu einer Vielzahl wichtiger Werke aus den Bereichen Wissenschaft, Geisteswissenschaften und Kunst. Bei JOSHA glauben wir, dass "Wissen, das nicht vermittelt wird, verlorenes Wissen ist". Deshalb ermutigen wir Studenten, Forscher und Künstler aus der ganzen Welt, ihre Arbeiten mit JOSHA zu veröffentlichen.

Unsere erste Ausgabe erschien im Dezember 2014 und inzwischen haben wir 5 Bände mit 17 Ausgaben und mehr als 250 Artikeln veröffentlicht (Mai 2018). Unsere Vertriebsgröße umfasst derzeit über 400.ooo Views und über 300.000 Downloads. Unsere Autoren und Leser reichen von Studenten und Doktoranden über Doktoranden bis hin zu Künstlern, Nobelpreisträgern und Wissenschaftlern. JOSHA ist auch in ROAD, e-journal.org und Scilit indiziert. Die Publikationssoftware JOSHA Journal wurde von der JOSHA GmbH Services entwickelt und an die International Academy of Sciences IASHA e.V. lizenziert. Wir danken auch der Biothera-Roland Mertelsmann Stiftung für die großzügige Unterstützung, die es uns ermöglicht, weiterhin qualifizierte Beiträge kostenlos zu veröffentlichen. 


\section{Internationale Akademie der Wissenschaften, Geisteswissenschaften und Künste}

(www.iasha.org)

Die International Academy of Sciences and Arts (IASHA) ist ein gemeinnütziger Verein, der sich der Förderung von Wissenschaft und Kunst und ihrem

interdisziplinären Austausch verschrieben hat. Im Mittelpunkt der Akademie steht die Förderung des wissenschaftlichen und künstlerischen Nachwuchses.

Dies geschieht durch:

- die Organisation von Symposien

- die Vergabe von Stipendien und Zuschüssen

- JOSHA, eine Zeitschrift mit offenem Zugang zum Internet, die multidisziplinäre und sprachlich unabhängige wissenschaftliche und künstlerische Arbeiten veröffentlicht.

Der Verein mit Sitz in Freiburg in Brisgovia wurde 2015 mit dem Ziel gegründet, den

Wissensaustausch zwischen verschiedenen wissenschaftlichen und künstlerischen Disziplinen zu erleichtern und Studierende sowie junge Wissenschaftler und Künstler zu unterstützen.

Die Publikation von JOSHA, das Journal of Sciences, Humanities and the Arts (www.josha-journal.org), spielt eine wichtige Rolle und enthält die folgenden Grundgedanken:

- Wissen, das nicht kommuniziert / veröffentlicht wird, ist verlorenes Wissen.

- Multidisziplinär, mehrsprachig und frei zugänglich.

- Internetbasierte Lehrbücher für Länder mit niedrigem Einkommen.

- Globale Kommunikation ohne sprachliche Einschränkungen.

Das internationale Team wird von Prof. em. Dr. Drs. H.c. Roland Mertelsmann und Prof. Dr. med. Gerhard Steinmann aus den verschiedenen wissenschaftlichen Fakultäten und Förderern zusammen.

Vorteile für Mitglieder und andere:

- Abonnement von JOSHA, regelmäßige Informationen über die neuesten Veröffentlichungen bei JOSHA.

- Einladungen zu exklusiven Veranstaltungen.

- Eigene Publikationen in JOSHA sind gebührenfrei.

- Persönliche Kontakte zu Wissenschaftlern und Gewinnern. 
IASHA Board of Directors

Prof. Dr. Gerhard Steinmann, CEO

Prof. em. Dr. Drs. H.c.c.c. Roland Mertelsmann,

Vizepräsident des Vorstands

Dr. Stephan Seiler, Geschäftsführer

Christiane Lange, Schatzmeisterin

Kontakt

Bernardin Dörr

bernardin.doerr@josha-archive.org

T: +4976142961090

Bank

Eigentümer: IASHA e.V.

Bank: Sparkasse Freiburg Nördl. breisgau

IBAN: DE19 6805 01010013583583

BIC: FRSPDE66XXX

PayPal: iasha.e.v.donate@gmail.com

Adresse

IASHA e.V.

c / o Prof. G. Steinmann Amtsgericht Fribourg

Talaeckerweg 3 VR 701346

D-89155 Erb 